\title{
Alpha-Dihydroergocryptine vs. Pramipexole as Adjunct Symptomatic Treatment of Idiopathic Parkinson's
}

\author{
Ulises Rodríguez Ortiz', Daniel San-Juan1, Francesco Scarci² \\ ${ }^{1}$ Movement Disorders Clinic and Neurophysiology Department, Instituto Nacional de Neurología y Neurocirugía, \\ Mexico City, Mexico \\ ${ }^{2}$ Scientific Department, Polichem S.A., Lugano, Switzerland \\ Email: francesco.scarci@polichem.com
}

Received 15 November 2014; revised 17 December 2014; accepted 5 January 2015

Copyright (C) 2015 by authors and Scientific Research Publishing Inc.

This work is licensed under the Creative Commons Attribution International License (CC BY). http://creativecommons.org/licenses/by/4.0/

(c) (i) Open Access

\section{Abstract}

A randomized, double blind, and active reference-controlled study was carried out among 116 patients suffering from idiopathic Parkinson's disease (PD). The aim of the study was to compare the safety and efficacy of alpha-dihydroergocryptine (DHEC) vs. pramipexole (PRAM) as an adjunct symptomatic therapy to levodopa in PD patients. The motor symptoms, assessed by the Unified Parkinson's Disease Rating Scale (UPDRS) III subscale, was identified as efficacy target. Fifty-six patients were randomized to DHEC and 60 to PRAM. Patients included were under constant levodopa dose for at least 3 months before entering the study, with baseline UPDRS III $\geq 14$. They underwent a 16-week treatment. Out of the 116 included patients, 85 (39 in DHEC group and 46 in PRAM group, respectively) completed the study protocol. In DHEC group, UPDRS III decreased by $\mathbf{2 4 . 2 \%}$ from baseline at week 10 and by $28.1 \%$ at week 16. In PRAM group, UPDRS III decreased by $27.1 \%$ from baseline at week 10 and by $29.2 \%$ at week 16 . The data were highly significant ( $p$ < 0.01 ) at each time point versus baseline, while no significant difference was noticed between treatments. Overall, the patient population did not show any clinically meaningful mood disturbances at baseline and the fluctuations of UPDRS I during treatment were devoid of clinical significance. Safety was fairly good in both groups. In conclusion, DHEC and PRAM proved to be effective and safe as adjunct therapy to levodopa in idiopathic PD. According to the research result, they have significantly improved the motor function of our patients.

\section{Keywords}

Adjunct Symptomatic Therapy, Alpha-Dihydroergocryptine, Pramipexole, Idiopathic Parkinson's Disease, Motor Complications, UPDRS 


\section{Introduction}

Since a long time, no new therapies on PD have been developed, with the exception of surgical measures limited to few severely ill and complicated patients. Dopamine agonists still provide an important option in the treatment of PD, as motor fluctuations and dyskinesia are less common with these medications [1]-[3] than that with levodopa. Pramipexole (PRAM) is a dopamine D3 agonist which has showed benefit to PD patients both as a monotherapy and as an adjunct therapy to levodopa. According to the Evidence-based medical (EBM) review update of 2005 [4], PRAM was judged to represent an effective tool in symptomatic monotherapy and adjunct to levodopa therapy, as well as in prevention and treatment of motor complications in PD. Adverse effects include hallucinations, edema, sudden sleep attacks, pathological gambling and impulsive control disorders. Its safety is defined as acceptable risk, nevertheless therapeutic alternatives may be needed in a non-negligible percentage of patients.

Ergot derivatives still represent an alternative in the symptomatic treatment of PD, but pergolide and cabergoline cause a heart valve fibrosis in long term treatment, relegating these two drugs to a secondary role in the management of PD [5]. Dihydroergocryptine (DHEC) is a hydrogenated ergot derivative with agonist activity on dopamine D2 receptors, which has been reported as an effective drug in symptomatic monotherapy of PD [4] [6], while there are fewer but promising data [7] on the symptomatic efficacy as an adjunct treatment to levodopa and treatment of motor complications. DHEC has been reported as very safe; no valvular fibrosis, sudden sleep or pathological gambling have ever been reported with this drug, as granted even by EMA [8].

DHEC was reported to be as effective as bromocriptine [9] on motor symptoms and more effective than lisuride [7] on fluctuations, but better tolerated. Further studies are needed to confirm whether this drug may be really an alternative to PRAM even as adjunct therapy to levodopa. As no comparative studies between DHEC and PRAM have ever been published, the aim of our study was to investigate the efficacy and safety of the two drugs as an adjunct therapy to levodopa in idiopathic PD.

\section{Material \& Methods}

The study followed a randomized, parallel groups, double blind and prospective design. Before the start of the study, the protocol was approved by the local Bioethics Committee. The study was performed between 2008 and 2010 at Instituto Nacional de Neurología y Neurocirugía, Mexico City, according to ICH GCP guidelines. Signed informed consent was obtained from all patients prior to start any protocol procedure.

Inclusion Criteria: patients of both genders with idiopathic PD, according to the UK Brain Bank criteria at constant levodopa dose $\leq 1500 \mathrm{mg}$ daily, Eligible patients had Hoehn \& Yahr stage (HYS) $\geq 1$, UPDRS III $\geq 14$, age 40 - 70, and had had constant levodopa dose for at least 3 months. Exclusion criteria: presence of cerebral micro-vessel injury, patients having previously undergone surgical procedures for PD, metabolic diseases, kidney or liver diseases, psychiatric disturbances, history of acute myocardial infarction.

Patients were randomly assigned either to dihydroergocryptine (Diamin ${ }^{\circledR}$, Laboratorios Grossman, Mexico) (DHEC) or to pramipexole (Miropex ${ }^{\circledR}$, Boehringer-Ingelheim, México) (PRAM). To assure the double blind, the secondary packaging of the treatments were matching and the information on the treatment was not available either to the patient or to the Principal Investigator, who was responsible for the allocation of the treatment as well as for the efficacy and safety evaluations.

In both treatment groups, the daily dosage was gradually increased to reach the target dose as per the approved labelling and in accordance with previous literature [10] [11].

During the whole study, the levodopa dosage was kept as constant as possible at the dose level of the study entry. The need to increase levodopa to treat PD symptoms worsening was judged as a negative efficacy endpoint, and the levodopa dose reduction, due to manifest PD symptoms improvement, was judged as a positive efficacy endpoint. Levodopa dose variation was also allowed in case of any serious or severe adverse event. Both levodopa + carbidopa (Sinemet $^{\circledR}$, Merck sharp \& Dohme, Mexico) or levodopa + benserazide (Madopar ${ }^{\circledR}$, Roche, Mexico) combinations were allowed, no combination with entacapone was permitted either at the enrolment or during the whole study duration. No other anti-Parkinson treatment was permitted at the study entry. Anticholinergic agents had to be withdrawn 5 days before enrolment. Only if needed due to insufficient symptom control, patients were allowed to take biperiden (max $8 \mathrm{mg}$ daily) or trihexyphenidyl (max $15 \mathrm{mg}$ daily). Sedative or hypnotics had to be withdrawn at least 2 weeks before study entry and preferably they had to be avoided during the study. All dopamine and serotonin agonist and antagonists other than the study drugs were 
not permitted throughout the study.

Baseline evaluations of the illness severity included UPDRS I-IV, Hoehn \& Yahr staging and Schwab \& England scale (S \& E). Laterality, specific motor and non-motor symptoms and ancillary symptoms were also evaluated.

Co-primary efficacy variables were UPDRS total and UPDRS III subscale in "ON", namely motor symptoms, assessed at study entry (baseline) and after 4, 10 and 16 weeks (end of study). Secondary efficacy variables consisted of UPDRS I, II and IV. Safety was evaluated by analyzing vital signs, blood tests and blood chemistry, urine tests and the occurrence of any adverse events at each visit. Descriptive statistics were provided on demographic and baseline values, and homogeneity between treatments was tested by means of the chi-square test $\left(\chi^{2}\right)$ or t-test on unadjusted UPDRS values. Descriptive statistics of levodopa dose between groups at each time point have been also provided. The change from baseline for each part of the UPDRS to visit at week 10 and week 16 were calculated as the difference of the baseline minus the actual value. Efficacy on primary and secondary parameters was assessed between treatments using a Mixed-Effect Model Repeated Measure (MMRM) with the change from baseline at each visit as dependent variable, treatment, visit, treatment $\mathrm{x}$ visit interaction as fixed effects and baseline as covariates. Vital signs, including heart rate, respiratory rate and blood pressure, were tested by Student t-test for independent data between treatments at baseline and end of study and for paired data between times within treatment group.

\section{Results}

Overall, 116 patients entered the study randomly assigned to DHEC (56) and to PRAM (60). At baseline, the two groups were homogeneous with regards to demographic and clinical characteristics (see Table 1).

The number of patients who prematurely terminated the study was 17 in DHEC group and 14 in PRAM group (Table 2). Overall, 85 patients completed the study protocol and form the PP population, namely 39 (20 males and 19 females, mean age $60.35 \pm 8.9$ years) in DHEC group and 46 (30 males and 16 females, mean age $58.52 \pm$ 9.49 years) in PRAM group, respectively.

In the PP population, main symptom at entry in DHEC group was tremor (43.6\%), followed by rigidity (28.2\%) and bradykinesia (28.2\%). In PRAM group, main symptom was tremor (56.5\%), followed by rigidity (23.9\%) and bradykinesia (21.7\%) (not significant between treatments). Motor fluctuations were present in 76.9\% of patients for DHEC and $80.0 \%$ for PRAM, respectively. Unilateral PD was present in $74.4 \%$ (DHEC) and $86.7 \%$ (PRAM). Bilateral PD was present in $25.6 \%$ and $13.3 \%$, respectively. The most affected side was the right one in 22 patients for DHEC, 21 for PRAM, the left one for 11 patients (DHEC) and 20 (PRAM).

In the primary parameter, the two groups were in general homogeneous at baseline, with a slightly more severe index of motor symptoms in DHEC: UPDRS III $32.08 \pm 13.89$ compared to $30.67 \pm 13.87$ in PRAM.

Depressive, cognitive impairment and behavior disturbance features were observed in both groups, with UPDRS I $2.38 \pm 1.664$ in DHEC and $2.28 \pm 2.105$ in PRAM. Activities of daily living (UPDRS II) were $12.87 \pm$ 5.70 for DHEC and $14.11 \pm 7.75$ for PRAM. Motor complications, such as fluctuation, dyskinesia, scored at the UPDRS IV $5.41 \pm 3.60$ for DHEC and $6.00 \pm 3.67$ for PRAM. The total UPDRS was at baseline $52.74 \pm 19.85$ in DHEC and $53.07 \pm 22.43$ for PRAM, none of the above differences being statistically significant.

HYS at baseline showed a median of stages 2, 2.5 and 3 in both treatment groups. S \& E activities of daily living indicated that patients were completely independent in the vast majority of cases. Details are reported in Table 1.

In DHEC, levodopa dose was increased in two patients from $500 \mathrm{mg}$ at baseline to $750 \mathrm{mg}$ from week 10 . The dose was decreased in other 2 patients, from 500 to 375 from week 10, and from 750 to $500 \mathrm{mg}$ from week 16 . In PRAM, levodopa dose was decreased in 3 patients from week 10 from 1000 to 750 , from 875 to 750 and from 375 to $200 \mathrm{mg}$, but in the last 2 patients it was increased again to basal dose from week 16. Levodopa dose throughout the study is summarized in Table 3. There were no significant differences between times in either group.

UPDRS total score improved in both treatment groups at all-time points versus baseline $(\mathrm{P}<0.01)$. At the end of treatment, UPDRS total scored $42.15 \pm 18.24$ in DHEC and $38.22 \pm 18.30$ in PRAM (the differences versus baseline were not significant between treatments).

Motor examination assessed by UPDRS III improved with a final/baseline decrease of $28.15 \%$ in DHEC, and of 29.25\% in PRAM: P < 0.01 between times at week 10 and week 16, not significant between treatments. Details of the co-primary efficacy endpoints are reported in Table 4. 
Table 1. Demographic and clinical characteristics of patients at baseline (safety population).

\begin{tabular}{|c|c|c|}
\hline & Dihydroergocryptine & Pramipexole \\
\hline No. of patients and gender $(\mathrm{M} / \mathrm{F})$ & $56(29 / 27)$ & $60(36 / 24)$ \\
\hline Age (years) (mean \pm SD) & $59.18 \pm 9.74$ & $58.17 \pm 9.57$ \\
\hline \multicolumn{3}{|l|}{ Main sign } \\
\hline Tremor & $30(53.6 \%)$ & $39(65.0 \%)$ \\
\hline Bradykinesia & $10(17.9 \%)$ & $8(13.3 \%)$ \\
\hline Rigidity & $16(28.6 \%)$ & $13(21.7 \%)$ \\
\hline \multicolumn{3}{|l|}{ Hoehn \& Yahr stage (n, \%) } \\
\hline Stage 1 & $1(1.8 \%)$ & $4(6.6 \%)$ \\
\hline Stage 1.5 & $3(5.4 \%)$ & $5(8.3 \%)$ \\
\hline Stage 2 & $27(48.2 \%)$ & $20(33.3 \%)$ \\
\hline Stage 2.5 & $12(21.4 \%)$ & $14(23.3 \%)$ \\
\hline Stage 3 & $9(16.1 \%)$ & $11(18.3 \%)$ \\
\hline Stage 4 & $4(7.1 \%)$ & $5(8.3 \%)$ \\
\hline Stage 5 & 0 & $1(1.7 \%)$ \\
\hline \multicolumn{3}{|l|}{ UPDRS (mean \pm SD) } \\
\hline Total score & $51.42 \pm 20.04$ & $49.98 \pm 20.34$ \\
\hline Mentation, behaviour and mood & $2.25 \pm 2.06$ & $2.46 \pm 2.02$ \\
\hline Activities of daily living & $14.25 \pm 6.78$ & $12.61 \pm 6.75$ \\
\hline Motor examination & $30.7 \pm 13.32$ & $30.86 \pm 13.54$ \\
\hline Treatment complications & $4.22 \pm 3.12$ & $4.05 \pm 3.14$ \\
\hline \multicolumn{3}{|l|}{ Schwab \& England scale (n, \%) } \\
\hline $10 \%$ & 0 & $1(1.7 \%)$ \\
\hline $20 \%$ & 1 (1.8\%) & 0 \\
\hline $30 \%$ & 0 & $1(1.7 \%)$ \\
\hline $40 \%$ & 1 (1.8\%) & 1 (1.7\%) \\
\hline $50 \%$ & 1 (1.8\%) & $1(1.7 \%)$ \\
\hline $60 \%$ & 2 (3.6\%) & $1(1.7 \%)$ \\
\hline $70 \%$ & $1(1.8 \%)$ & $5(8.3 \%)$ \\
\hline $80 \%$ & $13(23.2 \%)$ & 8 (13.3\%) \\
\hline $90 \%$ & 17 (30.4\%) & $15(25.0 \%)$ \\
\hline $100 \%$ & $20(35.7 \%)$ & 27 (45.0\%) \\
\hline
\end{tabular}

Table 2. Patient disposition and main cause of discontinuation.

\begin{tabular}{ccc}
\hline Number of Patients & Dihydroergocryptine & Pramipexole \\
\hline Randomized & 56 & 60 \\
Not included (consent withdrawal) & - & - \\
Safety population & 56 & 60 \\
Completed & 39 & 46 \\
Discontinued & 17 & 14 \\
PP population & 39 & 46 \\
\hline Main cause of discontinuation & & - \\
\hline Adverse event (nausea) & 2 & 8 \\
Abandon (lost to follow up) & 1 & 4 \\
Excluded & 12 & 2 \\
\hline
\end{tabular}


Table 3. Levodopa dose (mg daily) throughout the study. Data are means \pm SD.

\begin{tabular}{cccc}
\hline Treatment group & Baseline & Week 10 & Week 16 \\
\hline Dihydroergocryptine & $605.5 \pm 286.3$ & $615.4 \pm 289.6$ & $605.0 \pm 278.8$ \\
Pramipexole & $681.3 \pm 238.8$ & $669.3 \pm 287.6$ & $675.8 \pm 290.4$ \\
\hline
\end{tabular}

Table 4. Mean values of UPDRS at baseline and changes by Treatment andtimes (PP Population).

\begin{tabular}{|c|c|c|c|c|c|}
\hline & \multicolumn{2}{|c|}{ DHEC (n = 39) } & \multicolumn{2}{|c|}{ PRAM $(n=46)$} & \multirow{2}{*}{$\begin{array}{l}\text { t-test for } \\
\text { difference }\end{array}$} \\
\hline & Mean & Std & Mean & Std & \\
\hline UPDRS I at baseline & 2.38 & 1.66 & 2.28 & 2.10 & \\
\hline Change of UPDRS I to week 10 & 0.64 & 1.66 & 0.91 & 1.77 & 0.4705 \\
\hline Change of UPDRS I to week 16 & -0.05 & 1.34 & 0.80 & 1.76 & 0.0148 \\
\hline UPDRS II at baseline & 12.87 & 5.69 & 14.11 & 7.75 & \\
\hline Change of UPDRS II to week 10 & 1.13 & 3.93 & 3.54 & 5.26 & 0.0206 \\
\hline Change of UPDRS II to week 16 & 1.08 & 4.07 & 3.83 & 4.97 & 0.0071 \\
\hline UPDRS III at baseline & 32.08 & 13.89 & 30.67 & 13.87 & \\
\hline Change of UPDRS III to week 10 & 7.77 & 6.62 & 8.30 & 9.50 & 0.7681 \\
\hline Change of UPDRS III to week 16 & 9.03 & 8.15 & 8.98 & 11.56 & 0.9829 \\
\hline UPDRS IV at baseline & 5.41 & 3.60 & 6.00 & 3.67 & \\
\hline Change of UPDRS IV to week 10 & 0.62 & 2.85 & 1.37 & 2.92 & 0.2342 \\
\hline Change of UPDRS IV to week 16 & 0.54 & 2.70 & 1.24 & 2.89 & 0.2548 \\
\hline UPDRS total at baseline & 52.74 & 19.85 & 53.07 & 22.43 & \\
\hline Change of UPDRS total to week 10 & 10.15 & 10.33 & 14.13 & 14.21 & 0.1504 \\
\hline Change of UPDRS total to week 16 & 10.59 & 11.10 & 14.85 & 17.12 & 0.1861 \\
\hline
\end{tabular}

With reference to the secondary endpoints, the UPDRS I decreased in PRAM, while there was an irregular trend of changes in DHEC ( $\mathrm{P}=0.004$ between treatments). UPDRS II improved in both treatment groups with the significantly greater improvement in favor of PRAM: UPDRS II at the end of treatment, $11.79 \pm 6.08$ in DHEC and $10.28 \pm 5.86$ in PRAM. UPDRS IV improved in both groups at all-time points versus baseline. UPDRS IV at the end of treatment was $4.87 \pm 3.83$ in DHEC and $4.76 \pm 3.38$ in PRAM (not significant). Details of the secondary efficacy endpoint are reported in Table 4.

All randomized patients entered the safety analysis, i.e. 57 patients in DHEC and 60 in PRAM group, respectively. Overall, 3 patients complained of related adverse events during the first week, in DHEC group. At the recording of week 10, adverse events were complained of by 20 patients (33.3\%) in DHEC and 16 (26.7\%) in PRAM. At the 16-week visit, adverse events were complained of by 8 patients per group, corresponding to $14 \%$ of DHEC and $13.3 \%$ of PRAM patients. The side effects are displayed in Table 5.

The most frequent adverse reactions were on gastrointestinal tract for both treatments, with a slight prevalence for DHEC. Two patients prematurely stopped the treatment with DHEC due to severe nausea in early phases of treatment. No dropout was recorded in PRAM. Occurrence of motor symptoms and CNS disturbances was similar, but PRAM revealed a higher frequency of sleep disorders and CNS stimulation. In those patients who continued the treatment according to the study protocol, the side effects were tolerated and their frequency markedly decreased during the late stage of treatment. Differences between treatments were not significant.

\section{Discussion}

The existing literature [4] [12] of DHEC indicates that this drug is recognized as efficacious in PD monotherapy, while its efficacy as an adjunct therapy to levodopa is still considered to be investigational. As DHEC is en- 
Table 5. Adverse events classified by system organ class and preferred term by treatment group and time points (safety population).

\begin{tabular}{|c|c|c|c|c|c|c|}
\hline \multirow[t]{2}{*}{ SOC } & \multirow[t]{2}{*}{ PT } & \multicolumn{3}{|c|}{ DHEC $(n=56)$} & \multicolumn{2}{|c|}{ PRAM (n = 60) } \\
\hline & & Week 1 & Week 10 & Week 16 & Week 10 & Week 16 \\
\hline \multirow[t]{9}{*}{ Gastrointestinal disorders } & Nausea & 3 & 8 & 3 & 6 & 2 \\
\hline & Vomiting & & 2 & & & \\
\hline & Dry mouth & & 2 & & & \\
\hline & Salivation & & 1 & & & \\
\hline & Diarrhoea & & & & 1 & \\
\hline & Gastritis & & & 1 & 1 & \\
\hline & Constipation & & 1 & & 1 & \\
\hline & Heartburn & & 2 & & & \\
\hline & Abdominal pain upper & & 1 & & & \\
\hline $\begin{array}{c}\text { Metabolism and nutrition } \\
\text { disorders }\end{array}$ & Decreased appetite & 1 & 1 & & & \\
\hline \multirow[t]{2}{*}{$\begin{array}{l}\text { Musculoskeletal and } \\
\text { connective tissue disorders }\end{array}$} & Rigidity & & 3 & & 1 & \\
\hline & Pain in arm & & 1 & & & \\
\hline \multirow[t]{16}{*}{ Nervous system disorders } & Lower extremities weakness & 1 & & & & \\
\hline & Bradykinesia & & & & & 1 \\
\hline & Dyskinesia & & 3 & & 3 & 2 \\
\hline & Balance difficulty & 1 & & & & \\
\hline & Festinating gait & & & & 1 & \\
\hline & Headache & & 4 & 2 & & \\
\hline & Drowsiness & & 2 & & 1 & 1 \\
\hline & Insomnia & & & 1 & & \\
\hline & Vivid dreams & & & & & 1 \\
\hline & Confusion & & & & 1 & \\
\hline & Thirst & & & & 1 & \\
\hline & Asthenia & & & & 1 & \\
\hline & Flashing vision & 1 & & & & \\
\hline & Movement disorder & & 1 & & & \\
\hline & Parkinsonism aggravated & & & & 1 & \\
\hline & Dopamine dysregulation syndrome & & 1 & & & \\
\hline \multirow{3}{*}{$\begin{array}{l}\text { General disorders and } \\
\text { administration site } \\
\text { conditions }\end{array}$} & Fatigue & & 1 & & & \\
\hline & Lack of efficacy & & & & 1 & \\
\hline & Malaise & 1 & & & & \\
\hline $\begin{array}{l}\text { Respiratory, thoracic and } \\
\text { mediastinal disorders }\end{array}$ & Dyspnoea & & 1 & & & \\
\hline \multirow[t]{2}{*}{$\begin{array}{l}\text { Skin and subcutaneous } \\
\text { tissue disorders }\end{array}$} & Livedo reticularis & & & & & 1 \\
\hline & Skin rash & & & & 1 & \\
\hline
\end{tabular}




\begin{tabular}{|c|c|c|c|c|c|c|}
\hline \multicolumn{7}{|l|}{ Continued } \\
\hline & Hair loss & & & & & 1 \\
\hline Cardiac disorders & Tachycardia & 1 & & & & \\
\hline $\begin{array}{l}\text { Injury, poisoning and } \\
\text { procedural complications }\end{array}$ & Fall & & & & 2 & \\
\hline \multirow[t]{8}{*}{ Psychiatric disorders } & Irascibility & 1 & & & & \\
\hline & Expressive language disorder & & & & 1 & \\
\hline & Hallucination & & & 1 & & \\
\hline & Feeling of despair & & & 1 & & \\
\hline & Sleep disorders & & & & 1 & \\
\hline & Anxiety & & 1 & & & \\
\hline & Nervousness & & 1 & & & \\
\hline & Irritability & & & & 1 & \\
\hline
\end{tabular}

dowed with a characteristic pharmacological profile of dopamine D2 agonist, with an activity similar to bromocriptine but with better safety profile [9], this drug remains an important tool in the treatment of PD. Moreover, the pharmacovigilance enquiries carried out in recent years on all dopaminergic agents used to treat PD confirm that this drug does not share with D3 agonist agents the side effects on sleep and on pathological gambling [13], as well as not sharing the effect on cardiac valve fibrosis typical of pergolide and cabergoline [8].

Therefore, it seemed interesting to investigate in a controlled study the efficacy of DHEC as an adjunct to levodopa therapy of PD. PRAM was chosen as reference, as according to the EBM review [4], this drug represents an effective tool in symptomatic adjunct to levodopa therapy, as well as in prevention and treatment of motor complications.

The results obtained in our controlled study, confirm that DHEC may also be of benefit in patients with PD, already under stabilized levodopa treatment.

In our patients, both drugs induced a significant decrease of motor symptoms without any significant difference between treatments. Few levodopa dose adjustments happened in both groups and did not affect the efficacy results of the study. As expected, a large inter-individual variance was present at baseline and standard deviations were large (about one third of the means). Furthermore, as the protocol required to keep as constant as possible the levodopa dose across the study, it seemed more reasonable to discuss the individual dose changes rather than to perform an inferential analysis.

Only PRAM significantly decreased UPDRS I and II, while DHEC did not. This was not expected and may be due to the small number of patients included in our study.

Finally, our data show that both drugs had a similar effect on motor complications, with a significant decrease of UPDRS IV throughout the study (between times). There was a larger decrease in the score of this subscale with PRAM, of about $20 \%$, compared to $10 \%$ with DHEC, which accounted for a higher score in PRAM group at baseline. No statistically significant difference between treatments was found.

The treatment period of the study was too short to evaluate potential long term side effects of both drugs. In general, safety was good in this study: as expected, nausea was the most frequent side effect in both groups. DHEC, being mostly a D2 agonist agent, had more side effects on gastrointestinal tract, while PRAM, which is a D3 agonist, had more side effects on CNS, including anxiety/nervousness, irritability and thirst. Skin reactions were also noticed only in PRAM group.

While peripheral adverse drug reactions, such as nausea, vomiting or orthostatic hypotension, can be effectively treated and usually pose few problems to many patients, neuropsychiatric events can seriously limit the use of PRAM in some cases. Certain effects such as excessive daytime somnolence, impulse-control disorders, hallucinations or delusions were not seen in our short lasting study.

\section{Conclusion}

In conclusion, dihydroergocryptine appeared of benefit to PD patients in this controlled study and may be considered to be a useful alternative to pramipexole as an adjunct to levodopa symptomatic therapy. 


\section{Conflicts of Interest and Sources of Funding}

FS is an employee of Polichem SA. Polichem SA provided funding of $10 \%$ of study expenses. Polichem did not have any role in study design; in the collection, analysis, and interpretation of data; in the writing of the report; and in the decision to submit the paper for publication. The study was funded by Laboratorios Grossman Mexico (90\%).

\section{References}

[1] Stacy, M. and Galbreath, A. (2008) Optimizing Long-Term Therapy for Parkinson Disease: Levodopa, Dopamine Agonists, and Treatment-Associated Dyskinesia. Clinical Neuropharmacology, 31, 51-56. http://dx.doi.org/10.1097/WNF.0b013e318065b088

[2] Hauser, R.A., Rascol, O., Korczyn, A.D., et al. (2007) Ten-Year Follow-Up of Parkinson's Disease Patients Randomized to Initial Therapy with Ropinirole or Levodopa. Movement Disorders, 22, 2409-2417. http://dx.doi.org/10.1002/mds.21743

[3] The Parkinson Study Group CALM Cohort Investigators (2009) Long-Term Effect of Initiating Pramipexole vs Levodopa in Early Parkinson Disease. Archives of Neurology, 66, 563-570. http://dx.doi.org/10.1001/archneurol.2009.32

[4] Goetz, C.G., Poewe, W., Rascol, O. and Sampaio, C. (2005) Evidence-Based Medical Review Update: Pharmacological and Surgical Treatments of Parkinson's Disease: 2001 to 2004. Movement Disorders, 20, 523-539. http://dx.doi.org/10.1002/mds.20464

[5] Andrejak, M. and Tribouilloy, C. (2013) Drug-Induced Valvular Heart Disease: An Update. Archives of Cardiovascular Diseases, 106, 333-339. http://dx.doi.org/10.1016/j.acvd.2013.02.003

[6] Bergamasco, B., Frattola, L., Muratorio, A., et al. (2000) Alpha-Dihydroergocryptine in the Treatment of de novo parkinsonian Patients: Results of a Multicentre, Randomized, Double-Blind, Placebo-Controlled Study. Acta Neurologica Scandinavica, 101, 372-380. http://dx.doi.org/10.1034/j.1600-0404.2000.90295a.x

[7] Battistin, L., Bardin, P.G., Ferro-Milone, F., et al. (1999) Alpha-Dihydroergocryptine in Parkinson’s Disease: A Multicentre Randomized Double Blind Parallel Group Study. Acta Neurologica Scandinavica, 99, 36-42. http://dx.doi.org/10.1111/j.1600-0404.1999.tb00655.x

[8] (2008) Questions and Answers on the Review of Ergot-Derived Dopamine Agonists, Ref. EMEA/CHMP/319054/2008.

[9] Bonuccelli, U., D’Antonio, P., D’Avino, C., et al. (1995) Dihydroergocryptine in the Treatment of Parkinson's Disease. Journal of Neural Transmission, 45, 239-245.

[10] Parkinson Study Group (1997) Safety and Efficacy of Pramipexole in Early Parkinson Disease. A Randomized DoseRanging Study. JAMA, 278, 125-130. http://dx.doi.org/10.1001/jama.1997.03550020057038

[11] Parkinson Study Group (2000) Pramipexole vs levodopa as initial treatment for Parkinson disease: A randomized controlled trial. JAMA, 284, 1931-1938. http://dx.doi.org/10.1001/jama.284.15.1931

[12] Rascol, O., Payoux, P., Ferreira, J. and Brefel-Courbon, C. (2002) The Management of Patients with Early Parkinson's Disease. Parkinsonism Relat Disord, 9, 61-67. http://dx.doi.org/10.1016/S1353-8020(02)00045-7

[13] MHRA Public Assessment Report (2007) Dopamine Agonists: Pathological Gambling and Increased Libido. 
Scientific Research Publishing (SCIRP) is one of the largest Open Access journal publishers. It is currently publishing more than 200 open access, online, peer-reviewed journals covering a wide range of academic disciplines. SCIRP serves the worldwide academic communities and contributes to the progress and application of science with its publication.

Other selected journals from SCIRP are listed as below. Submit your manuscript to us via either submit@scirp.org or Online Submission Portal.
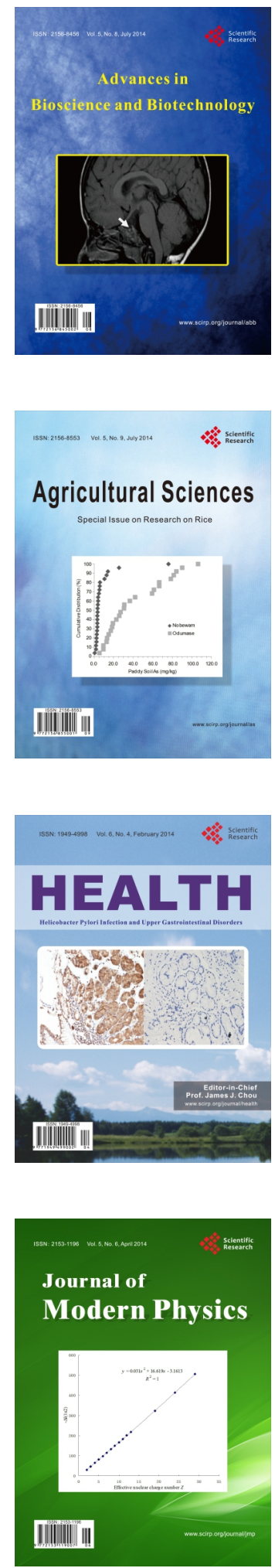
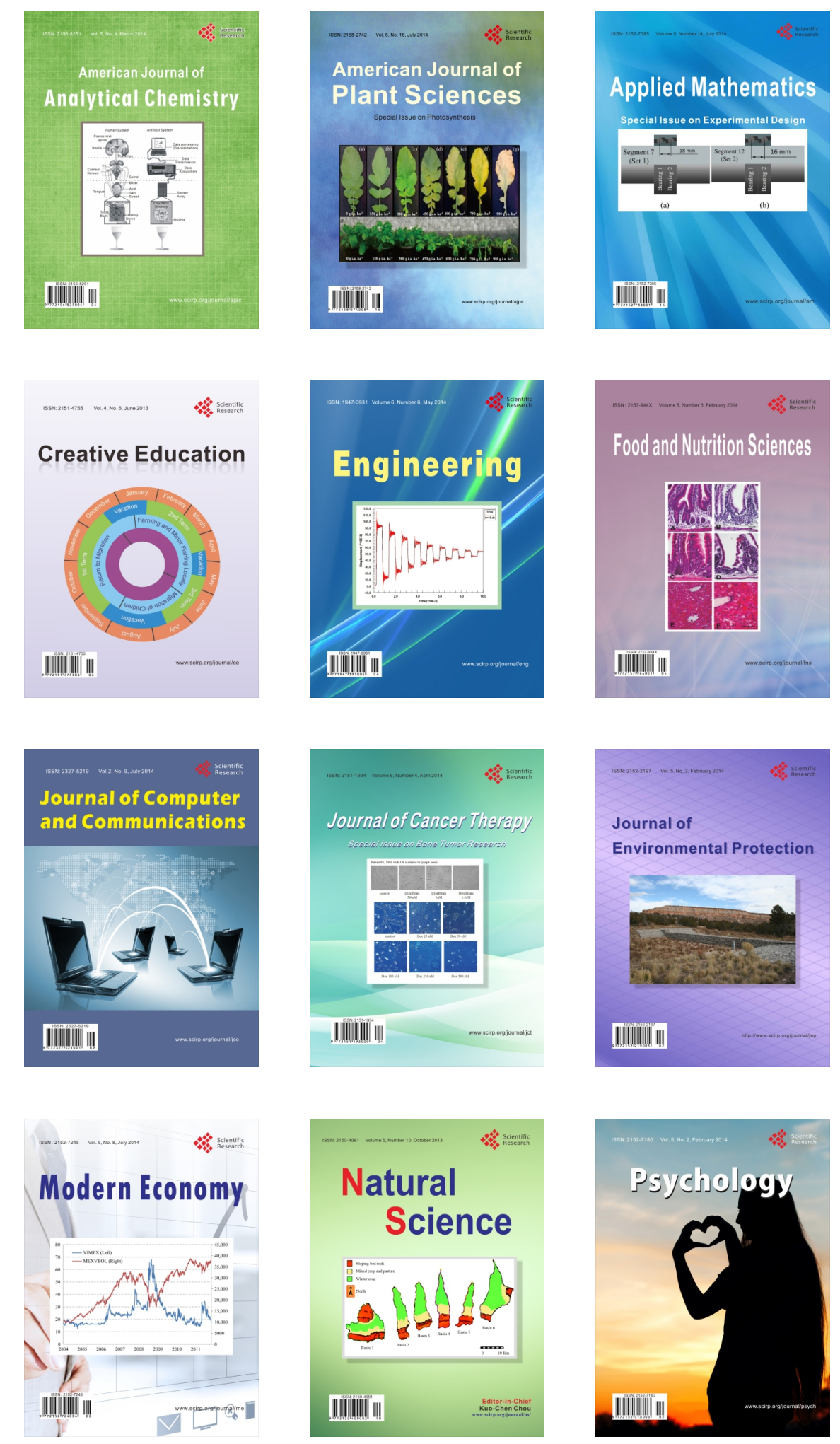